\title{
New Interpretation of the Concentration Dependence of the Compressibility of Aqueous Solutions of Nonelectrolytes
}

\author{
Jacek Gliński • Andrzej Burakowski
}

Received: 22 March 2010 / Accepted: 13 May 2010 / Published online: 1 June 2010

(C) The Author(s) 2010. This article is published with open access at Springerlink.com

\begin{abstract}
The parabolic-like plots of compressibility versus concentration of nonelectrolytes (ethanol as the example) in water are explained and described assuming the hydrate formation equilibrium reaction. Only two fitted parameters, the hydration number $n_{\mathrm{h}}$ and the cumulative formation constant of the hydrate $\beta_{n_{\mathrm{h}}}$, are used to reproduce the experimental data, including the minima of the adiabatic compressibility $\kappa_{S}$ versus composition at low solute contents. Another discussed option is a set of subsequent equilibrium reactions of hydration from which formation constants can also be determined. Calculations were performed for the water-ethanol system, and the results were compared with those obtained by a model recently developed by the authors.
\end{abstract}

Keywords Aqueous solutions · Compressibility $\cdot$ Hydrates $\cdot$ Nonelectrolytes

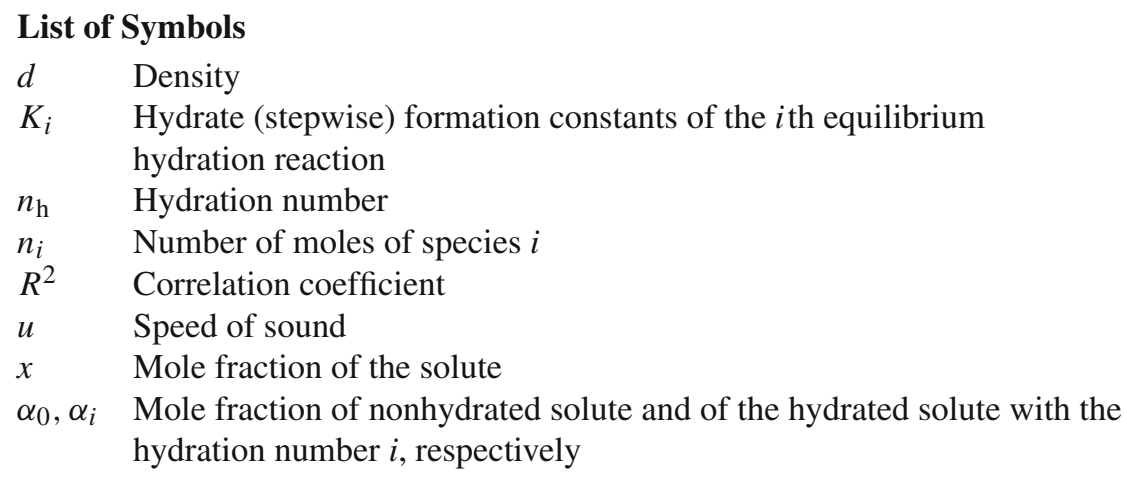

\footnotetext{
J. Gliński $(\bowtie) \cdot$ A. Burakowski

Faculty of Chemistry, University of Wrocław, F. Joliot-Curie 14, 50-383 Wrocław, Poland e-mail: glin@wchuwr.pl
} 
$\beta_{i} \quad$ Cumulative formation constant of the hydrate formation with the hydration (coordination) number $i$

$\kappa_{S} \quad$ Adiabatic compressibility coefficient of solution

$\kappa_{S, i} \quad$ Adiabatic compressibility coefficient of pure component $i$

\section{Introduction}

The adiabatic compressibility $\kappa_{S}$ can be calculated from the Laplace equation, $\kappa_{S}=$ $d^{-1} u^{-2}$ (where $d$ is the density, and $u$ is the speed of sound). This parameter is supposed to reflect the internal liquid structure and is known as an effective tool when investigating the structure of liquids and their intermolecular interactions. Note also that both the density and sound speed can be measured with very high accuracy of $0.01 \%$ or better.

When analyzing acoustic data in terms of liquid solution structure, usually simple models such as those based on the deviations of the measured parameters from less or more precisely defined additivity are applied [1]. However, these simple models fail when the solvent is water. The addition of minor amounts of solutes to water results in an initial linear decrease of the compressibility. This observation was the basis for the acoustic method of determination of hydration numbers derived by Pasynski [2]. The assumption is that water molecules involved in a hydrate are incompressible and that the compressibility of the bulk (nonhydrating) water is equal to that of the pure water solvent. The result is the following relation:

$$
n_{\mathrm{h}}=\frac{n_{\mathrm{H}_{2} \mathrm{O}}}{n_{\mathrm{X}}}\left(1-\frac{\kappa_{S}}{\kappa_{S, \mathrm{H}_{2} \mathrm{O}}}\right),
$$

where $n_{\mathrm{h}}$ is the hydration number, $n_{\mathrm{H}_{2} \mathrm{O}}$ and $n_{\mathrm{X}}$ are the numbers of moles of water and solute, respectively, in solution, and $\kappa_{S}$ and $\kappa_{S, \mathrm{H}_{2} \mathrm{O}}$ are, respectively, the compressibilities of the solution and of pure water. This equation was also applied by Pasynski [3] for nonelectrolytic solutions, and it was successfully exploited by us recently [4].

Aqueous nonelectrolyte solutions typically exhibit a maximum of the speed of sound (and the resulting minimum of compressibility) when plotted versus composition. It is necessary to add that the compressibility of pure water decreases with increasing temperature, which is anomalous behavior when compared to other liquids. This results in an interception of compressibility isotherms along the nonelectrolyte concentration in water. The composition corresponding to the interception point was attributed to the stoichiometry of labile clathrate-like hydrates which are forming in the solution [5]. A different interpretation is that of Baumgartner and Atkinson [6] who related this stoichiometry to the concentration of the minimum of the compressibility.

In this paper, our preliminary calculations are presented for the model which assumes formation of hydrates and needs only two parameters to be fitted: the association (hydration) number $n_{\mathrm{h}}$ and the equilibrium constant $\beta_{n_{\mathrm{h}}}$ corresponding to the formation of hydrates. This model was found to satisfactorily reproduce the experimentally observed, parabolic-like dependence of the compressibility versus concentration of ethanol in water. 


\section{Calculations}

The formation of a hydrate can be described in the same manner as is well known for coordination compounds. Two models were tested.

\subsection{Model of One Equilibrium Reaction Only (One-Constant Model)}

We consider the equilibrium state of the hydrate formation reaction:

$$
\mathrm{X}+n_{\mathrm{h}} \mathrm{H}_{2} \mathrm{O} \leftrightarrows \mathrm{X}\left(\mathrm{H}_{2} \mathrm{O}\right)_{n_{\mathrm{h}}}
$$

where $\mathrm{X}$ denotes a nonelectrolytic solute. This equilibrium can be described by its equilibrium constant $\beta_{n_{\mathrm{h}}}$ :

$$
\beta_{n_{\mathrm{h}}}=\frac{\left[\mathrm{X}\left(\mathrm{H}_{2} \mathrm{O}\right)_{n_{\mathrm{h}}}\right]}{[\mathrm{X}] \cdot\left[\mathrm{H}_{2} \mathrm{O}\right]^{n_{\mathrm{h}}}}
$$

The square brackets denote the molar concentration of species at equilibrium (more precisely, activities instead of molarities should be used). The value of $\left[\mathrm{H}_{2} \mathrm{O}\right]$ can be approximated by $c_{\mathrm{H}_{2} \mathrm{O}}-n_{\mathrm{h}} c_{\mathrm{X}}\left(c_{\mathrm{i}}\right.$ means the total, i.e., analytical molar concentration of component $i$ ). It can be found in any textbook that for a complex forming system is described by the above equilibrium (Eq. 2) where the equilibrium concentration of the free ligand (water in our case) is known; the mole fraction of the nonhydrated solute $\alpha_{0}$ and that of the hydrated solute $\alpha_{\mathrm{n}}$ are, respectively:

$$
\begin{aligned}
\alpha_{0} & =\frac{1}{1+\beta_{n_{\mathrm{h}}}\left[\mathrm{H}_{2} \mathrm{O}\right]^{n_{\mathrm{h}}}} \\
\alpha_{n} & =\frac{\beta_{n_{\mathrm{h}}}\left[\mathrm{H}_{2} \mathrm{O}\right]^{n_{\mathrm{h}}}}{1+\beta_{n_{\mathrm{h}}}\left[\mathrm{H}_{2} \mathrm{O}\right]^{n_{\mathrm{h}}}}
\end{aligned}
$$

Here the condition $\alpha_{0}+\alpha_{n}=1$ must be fulfilled. When the $\alpha$ 's are known, it is easy to calculate the equilibrium concentrations of all species existing in solution. Of course, the reverse situation is also possible, i.e., fitting the constant in a recurrence process.

\subsection{Model of Subsequent Equilibrium Reactions (Subsequent-Constants Model)}

This attempt assumes the formation of hydrates of different stoichiometry in stepwise formation reactions,

$$
\begin{aligned}
& \mathrm{X}+\mathrm{H}_{2} \mathrm{O} \leftrightarrows \mathrm{X}\left(\mathrm{H}_{2} \mathrm{O}\right) \\
& \mathrm{X}\left(\mathrm{H}_{2} \mathrm{O}\right)+\mathrm{H}_{2} \mathrm{O} \leftrightarrows \mathrm{X}\left(\mathrm{H}_{2} \mathrm{O}\right)_{2} \\
& \quad \quad \quad \\
& \mathrm{X}\left(\mathrm{H}_{2} \mathrm{O}\right)_{n_{\mathrm{h}}-1}+\mathrm{H}_{2} \mathrm{O} \leftrightarrows \mathrm{X}\left(\mathrm{H}_{2} \mathrm{O}\right)_{n_{\mathrm{h}}}
\end{aligned}
$$


where $n_{\mathrm{h}}$ means the maximum hydration number (maximum hydrate stoichiometry). The above equilibriums are described by their equilibrium constants,

$$
\begin{aligned}
K_{1} & =\frac{\left[\mathrm{X}\left(\mathrm{H}_{2} \mathrm{O}\right)\right]}{[\mathrm{X}] \cdot\left[\mathrm{H}_{2} \mathrm{O}\right]} \\
K_{2} & =\frac{\left[\mathrm{X}\left(\mathrm{H}_{2} \mathrm{O}\right)_{2}\right]}{\left[\mathrm{X}\left(\mathrm{H}_{2} \mathrm{O}\right)\right] \cdot\left[\mathrm{H}_{2} \mathrm{O}\right]} \\
& \cdots \\
K_{n_{\mathrm{h}}} & =\frac{\left[\mathrm{X}\left(\mathrm{H}_{2} \mathrm{O}\right)_{n_{\mathrm{h}}}\right]}{\left[\mathrm{X}\left(\mathrm{H}_{2} \mathrm{O}\right)_{n_{\mathrm{h}}-1}\right] \cdot\left[\mathrm{H}_{2} \mathrm{O}\right]}
\end{aligned}
$$

Instead of the formation constants $K_{\mathrm{i}}$, the cumulative ones are usually used, defined as $\beta_{1}=K_{1}, \beta_{2}=K_{1} K_{2}, \ldots, \beta_{n_{\mathrm{h}}}=K_{1} K_{2} \cdot \ldots \cdot K_{n_{\mathrm{h}}}$.

In this model, the solute exists in solution in different forms. By analogy to the former model, the molar fractions of these forms are, respectively,

$$
\begin{aligned}
\alpha_{0}= & \frac{1}{1+\beta_{1} \cdot\left[\mathrm{H}_{2} \mathrm{O}\right]+\beta_{2} \cdot\left[\mathrm{H}_{2} \mathrm{O}\right]^{2}+\cdots+\beta_{n_{\mathrm{h}}} \cdot\left[\mathrm{H}_{2} \mathrm{O}\right]^{n_{\mathrm{h}}}} \\
\alpha_{1}= & \frac{\beta_{1} \cdot\left[\mathrm{H}_{2} \mathrm{O}\right]}{1+\beta_{1} \cdot\left[\mathrm{H}_{2} \mathrm{O}\right]+\beta_{2} \cdot\left[\mathrm{H}_{2} \mathrm{O}\right]^{2}+\cdots+\beta_{n_{\mathrm{h}}} \cdot\left[\mathrm{H}_{2} \mathrm{O}\right]^{n_{\mathrm{h}}}} \\
\alpha_{2}= & \frac{\beta_{2} \cdot\left[\mathrm{H}_{2} \mathrm{O}\right]^{2}}{1+\beta_{1} \cdot\left[\mathrm{H}_{2} \mathrm{O}\right]+\beta_{2} \cdot\left[\mathrm{H}_{2} \mathrm{O}\right]^{2}+\cdots+\beta_{n_{\mathrm{h}}} \cdot\left[\mathrm{H}_{2} \mathrm{O}\right]^{n_{\mathrm{h}}}} \\
\alpha_{n_{\mathrm{h}}}= & \frac{\beta_{n_{h}} \cdot\left[\mathrm{H}_{2} \mathrm{O}\right]^{n_{\mathrm{h}}}}{1+\beta_{1} \cdot\left[\mathrm{H}_{2} \mathrm{O}\right]+\beta_{2} \cdot\left[\mathrm{H}_{2} \mathrm{O}\right]^{2}+\cdots+\beta_{n_{\mathrm{h}}} \cdot\left[\mathrm{H}_{2} \mathrm{O}\right]^{n_{\mathrm{h}}}}
\end{aligned}
$$

Again, the condition $\alpha_{0}+\alpha_{1}+\alpha_{2}+\cdots+\alpha_{n_{\mathrm{h}}}=1$ should be fulfilled. In this model, one fits $n_{\mathrm{h}}$ constants $\left(\beta_{1}, \beta_{2}, \ldots \beta_{n_{\mathrm{h}}}\right)$, where the maximum hydration number $n_{\mathrm{h}}$ is found by a trial-and-error procedure, fixing the subsequent integer values of $n_{\mathrm{h}}$ until a satisfactory quality of curve fitting is achieved.

Let us assume that solvates as a whole are incompressible $\left(\kappa_{S}=0\right)$, independent of their stoichiometry. As already mentioned in Sect. 1, a similar assumption was first made by Pasynski [2] and has been generally accepted in molecular acoustics.

The second assumption is that the compressibility of liquid mixtures is a linear function of the composition expressed in terms of mole fractions. In light of the above assumptions and the simple stoichiometric considerations, this can be written as follows:

$$
\kappa_{S}=\alpha_{0} x \kappa_{S, \mathrm{X}}+\left(1-x-n_{\mathrm{h}} \alpha_{\mathrm{n}} x\right) \kappa_{S, \mathrm{H}_{2} \mathrm{O}}
$$

where $x$ is the mole fraction of the solute, and $\kappa_{S, \mathrm{X}}$ and $\kappa_{S, \mathrm{H}_{2} \mathrm{O}}$ denote adiabatic compressibility coefficients of the pure solute $\mathrm{X}$ and water, respectively. 
Only the values of equilibrium constant(s) $\beta_{i}$ (for values of $n_{\mathrm{h}}$ fixed as integer numbers and changed stepwise until satisfactory fitting was achieved) were fitted in both the models tested. A commercial datasheet was sufficient for performing the calculations. For the first model, there are two fitted parameters, $n_{\mathrm{h}}$ and $\beta_{n_{\mathrm{h}}}$, but the former is determined through a trial-and-error process, and the latter by the classical least-squares method. In the second model, the stepwise constants $\beta_{\mathrm{i}}$ are determined in a similar way. As already mentioned, the experimental data for compressibilities of ethanol-water mixtures were taken from our recent paper [7].

\section{Results}

Data on the compressibilities of aqueous solutions of ethanol at $25^{\circ} \mathrm{C}$ were taken from [7]. Table 1 shows the results of curve fitting together with the correlation coefficients $R^{2}$, while Fig. 1 illustrates them. Note also that fittings were performed only for low and medium concentrations (below a mole fraction of 0.27 ) mainly because of the assumption on using molar concentrations instead of activities, but the results in Fig. 1 are calculated for the whole range of compositions. Our goal was to reproduce the irregular, parabolic-like shape of the dependence of $\kappa_{S}$ on ethanol content in water, and this has been achieved very successfully. It is obvious that the model recently presented in [8] is much less precise than any of the variants of the present one.

\section{Discussion}

Recently, we published [8] the results of a similar calculation made for twenty aqueous non-electrolytic systems and explaining the parabolic-like shape of $\kappa_{S}$ versus composition in terms of an equilibrium hydrate formation reaction, very similar to that presented in this work. However, the method presented in [8] was based on a few rather questionable assumptions. One of them was assuming that the compressibility is additive with volume fractions of components, not the molar ones. Moreover, the calculations made there did not consider the loss of water caused by hydration from its total content.

Inspection of the results presented in Table 1 and illustrated in Fig. 1 leads to the following conclusions and observations:

1. A hydration number of ethanol equal to 3 is close to the value of 2.2 obtained by the Pasynski acoustical method [4]. The present calculations for $n_{\mathrm{h}}=2$ and two cumulative constants yield much worse fitting (see Fig. 1).

2. Hydrates are formed in the equilibrium reaction (Eq. 2). This idea was already exploited and is reasonable [9].

3. The quality of fitting is the best for the subsequent model with three constants, but for the one-constant one, it is also very good. The two-constant model yields only semiquantitative agreement with the experimental data. The model presented in [8] (additivity of compressibility with volume fractions) describes very well the 


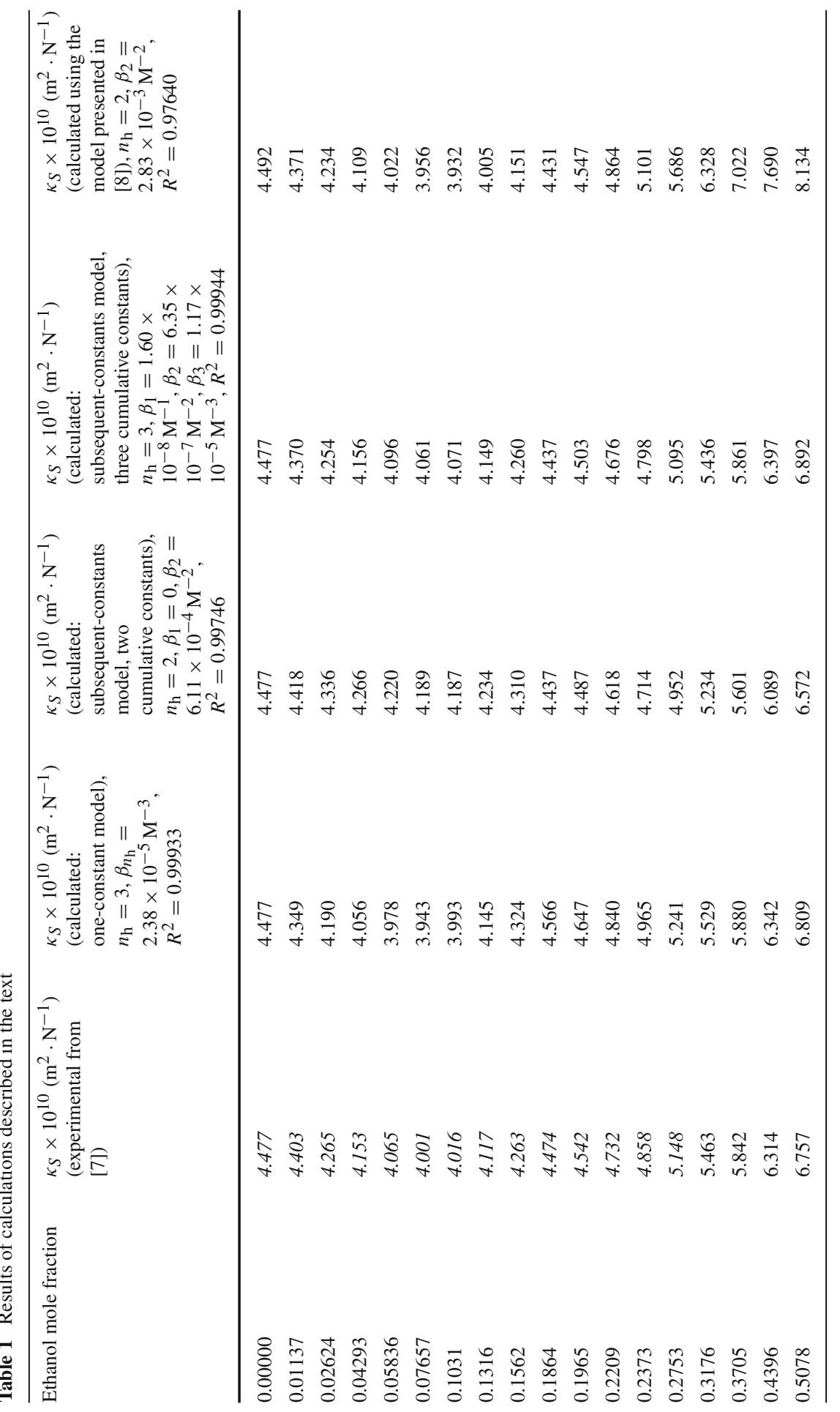




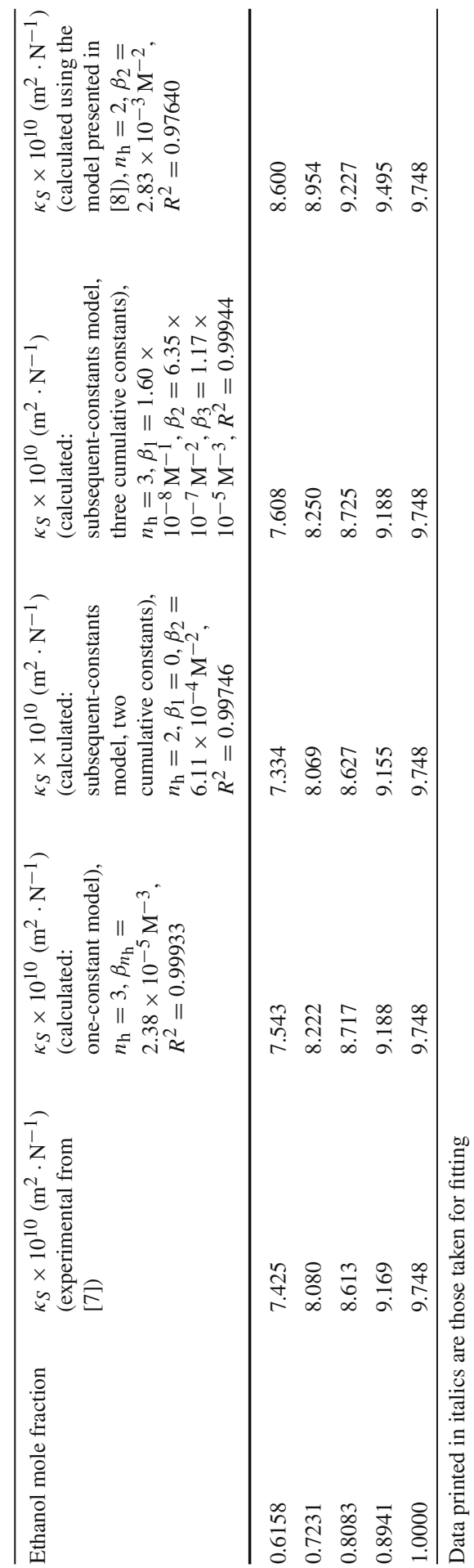




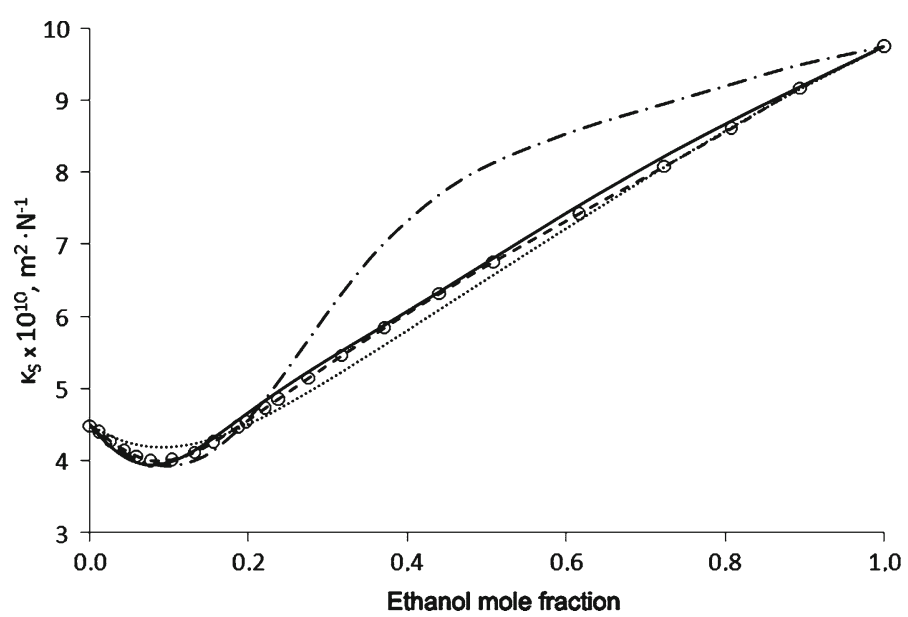

Fig. 1 Dependence of adiabatic compressibility coefficient $\kappa_{S}$ on mole fraction of ethanol in water at $25^{\circ} \mathrm{C}$. Experimental data from [7] (circles), fitted using one-constant model $\left(n_{\mathrm{h}}=3, \beta_{3}=2.38 \times 10^{-5} \mathrm{M}^{-3}\right.$, $R^{2}=0.99933$ ) (solid line), fitted using subsequent-constants model with two cumulative constants $\left(\beta_{1}=\right.$ $\left.0, \beta_{2}=6.11 \times 10^{-4} \mathrm{M}^{-2}, R^{2}=0.99746\right)$ (dotted line), fitted using subsequent-constants model with three cumulative constants $\left(\beta_{1}=1.60 \times 10^{-8} \mathrm{M}^{-1}, \beta_{2}=6.35 \times 10^{-7} \mathrm{M}^{-2}, \beta_{3}=1.17 \times 10^{-5} \mathrm{M}^{-3}\right.$, $\left.R^{2}=0.99944\right)$ (broken line), fitted results taken from $[8]\left(n_{\mathrm{h}}=2, \beta_{2}=2.83 \times 10^{-3} \mathrm{M}^{-2}, R^{2}=0.97640\right)$ (broken-dotted line)

real values only in the range of low ethanol contents. The present model is free of this problem although it also used only the data for low $x_{\text {ethanol }}$ in the curve fitting.

4. For the water-ethanol system, it is difficult to distinguish if only hydrates of stoichiometry $\mathrm{X}\left(\mathrm{H}_{2} \mathrm{O}\right)_{n_{\mathrm{h}}}$ are formed and consequently, the system can be described by either two parameters $n_{\mathrm{h}}$ and $\beta_{n_{\mathrm{h}}}$ only, or the subsequent three-constant model to better describe the equilibria. Calculations for many different systems could help in answering this question.

5. The equilibrium constants determined for the one-constant model $\left(n_{\mathrm{h}}=3, \beta_{n_{\mathrm{h}}}=\right.$ $\left.2.38 \times 10^{-5} \mathrm{M}^{-3}\right)$ and the three-constant model $\left(\beta_{3}=1.17 \times 10^{-5} \mathrm{M}^{-3}\right.$, two preceding constants are lower and have less influence on fitting results) are close enough to one another to expect that our calculations have deep physical sense.

In the literature, there are no data for the equilibrium constants of hydrate formation reactions for nonelectrolytes, except in a paper by Stokes and Robinson [10] who found for sucrose and glucose, respectively, $n_{\mathrm{h}}=11$ and 6 , and $K=0.994$ and 0.786, assuming that all the stepwise hydration constants are equal. However, simplifications made by them were too many and make their results less reliable. Thus, our attempt is pioneering work in determining these constants.

In conclusion, it should be stressed that the aqueous solutions of ethanol can be easily described by a very simple model, assuming formation of either one type of hydrate or a subsequent series of hydrates. The model reproduces the experimental $\kappa_{S}=f\left(x_{\text {ethanol }}\right)$ dependence with very good precision comparable to the recently 
published one [8]. However, the idea presented here should be tested for different systems. This will be the subject of our future papers.

Open Access This article is distributed under the terms of the Creative Commons Attribution Noncommercial License which permits any noncommercial use, distribution, and reproduction in any medium, provided the original author(s) and source are credited.

\section{References}

1. J. Gliński, J. Solution Chem. 31, 59 (2002)

2. A. Pasynski, Acta Physicochim. URSS 8, 385 (1938)

3. A. Pasynski, Acta Physicochim. URSS 22, 137 (1947)

4. A. Burakowski, J. Gliński, Chem. Phys. 332, 336 (2007)

5. H. Endo, Bull. Chem. Soc. Jpn 16, 1586 (1973)

6. E.K. Baumgartner, G.J. Atkinson, J. Phys. Chem. 75, 2336 (1971)

7. K. Jerie, A. Baranowski, S. Ernst, J. Gliński, Acta Phys. Polon. A 69, 81 (1986)

8. J. Gliński, A. Burakowski, J. Chem. Phys. 132, 124507 (2010)

9. F.J. Millero, A. Lo Surdo, C. Shin, J. Phys. Chem. 82, 784 (1978)

10. R.H. Stokes, R.A. Robinson, J. Phys. Chem. 70, 2126 (1966) 